\title{
Anabolic Steroid-Induced Myocardial Infarction in a Young Male
}

Fnu Samreen ${ }^{1}$, Ubaidullah Popal ${ }^{2}$, Zulfiqar A. Qutrio Baloch ${ }^{3}$

1. Cardiology, National Institute of Cardiovascular Diseases, Karachi, PAK 2. Internal Medicine, Tabba Heart Institute, Karachi, PAK 3. Cardiology, Sparrow Hospital, Lansing, USA

Corresponding author: Fnu Samreen, samreenmd20@gmail.com

\begin{abstract}
Misuse of androgenic-anabolic steroids (AAS) has been well known to increase the risk for a cardiac problem, including acute myocardial infarction (MI). Steroids once thought a magic drug providing immediate relief to patients, also have a darker aspect of its severe side effects. AAS are widely used these days, especially in teenagers, bodybuilders, and athletes. MI is thought to be a disease of old age, but young patients with MI without risk factors draw attention to the possibility of drugs such as cocaine, AAS abuse, and amphetamine.
\end{abstract}

In this article, we report the case of a 38-year-old African-American male, with a history of AAS abuse, who arrived at the emergency department with complaints of severe chest pain radiating to the left arm. An electrocardiogram (ECG) revealed ST-elevation MI (STEMI) and elevated troponin. The patient was transferred to the cardiac catheterization lab for an emergent catheterization which showed $100 \%$ stenosis of the left anterior descending artery and a drug-eluting stent was placed. An echocardiogram showed an ejection fraction of $35 \%$. All blood workup was negative. The patient was discharged on aspirin, ticagrelor, statin, ACE inhibitor, and B-blocker after three days.

Chest pain in a young patient population secondary to MI is not uncommon these days and the most important thing to evaluate is drug history, including AAS use. Athletes, bodybuilders, and others who use steroids or other drugs that are responsible for MI should be under the supervision of physicians so that the complications of steroids are ascertained, and if steroids are needed for any medical illness, proper dosage and follow-up should be emphasized. Therefore, while taking history from a patient, it is essential for physicians to be aware of this association of steroids with coronary artery disease.

Review began 01/13/2021 Review ended 01/24/2021 Published 02/01/2021

\section{(c) Copyright 2021}

Samreen et al. This is an open access article distributed under the terms of the Creative Commons Attribution License CC-BY 4.0., which permits unrestricted use, distribution, and reproduction in any medium, provided the original author and source are credited.
Categories: Cardiology

Keywords: anabolic steroid abuse, myocardial infarction, st-elevation myocardial infarction

\section{Introduction}

Risk factors for myocardial infarction (MI) in young people are highly significant and at this age drug abuse must always be considered. Athletes use androgenic-anabolic steroids (AAS) to increase their performance (protein synthesis in skeletal muscles is increased by AAS); however, there are critical adverse effects, including hepatic and endocrine dysfunction, and cardiovascular and behavioral changes have been reported [1].

Steroids have a broad spectrum of applications, ranging from treatment in medical emergencies and other diseases to abuse by athletes and other sports players. No doubt steroids have many good applications, but its overuse leads to serious consequences and sometimes even death [2].

An increased cardiovascular risk in those individuals who use these drugs has been shown [3]. ST-elevation MI (STEMI) is a life-threatening condition having 2.5-10\% mortality in the first month [4]. Conventional risk factors play an important role in coronary heart diseases, and nontraditional risk factors also need to be considered as they are present in more than $50 \%$ of coronary artery disease cases [5]. In a previous study, more than 150 drugs were reported as possible causes of MI, out of which 39 drugs were thought as the main suspects that can cause MI: prednisone, betamethasone, and dexamethasone [6].

\section{Case Presentation}

A 38-year-old African American male with no significant past medical history (he was using anabolic steroid [unknown duration] for muscle building and athlete) came to the emergency department with complaints of severe chest pain that started 30 minutes previously, which were crushing in nature, radiating to his left arm, and associated with sweating, nausea, and breathlessness. He denied similar pain in the past during exertion or rest. The patient smoked three cigarettes per day for 10 years. His family history was nonsignificant for ischemic heart disease. 


\section{Cureus}

On examination, the patient was sweaty, and vitals were: heart rate, 110 beats/min; blood pressure, 130/85 $\mathrm{mm} \mathrm{Hg}$; respiratory rate, 16/min; temp., 98.6 F [Normal 97-98 F]. His heart sounds were normal with no murmur. There was no lower extremity edema and normal peripheral pulses. A 12-lead electrocardiogram (ECG) showed ST-segment changes of the precordial leads of an acute anterior wall MI (Figure 1). The patient's serum creatine kinase and total cholesterol concentration were normal. Complete blood count, urea, creatinine, urine analysis, and urine drug screen were normal.

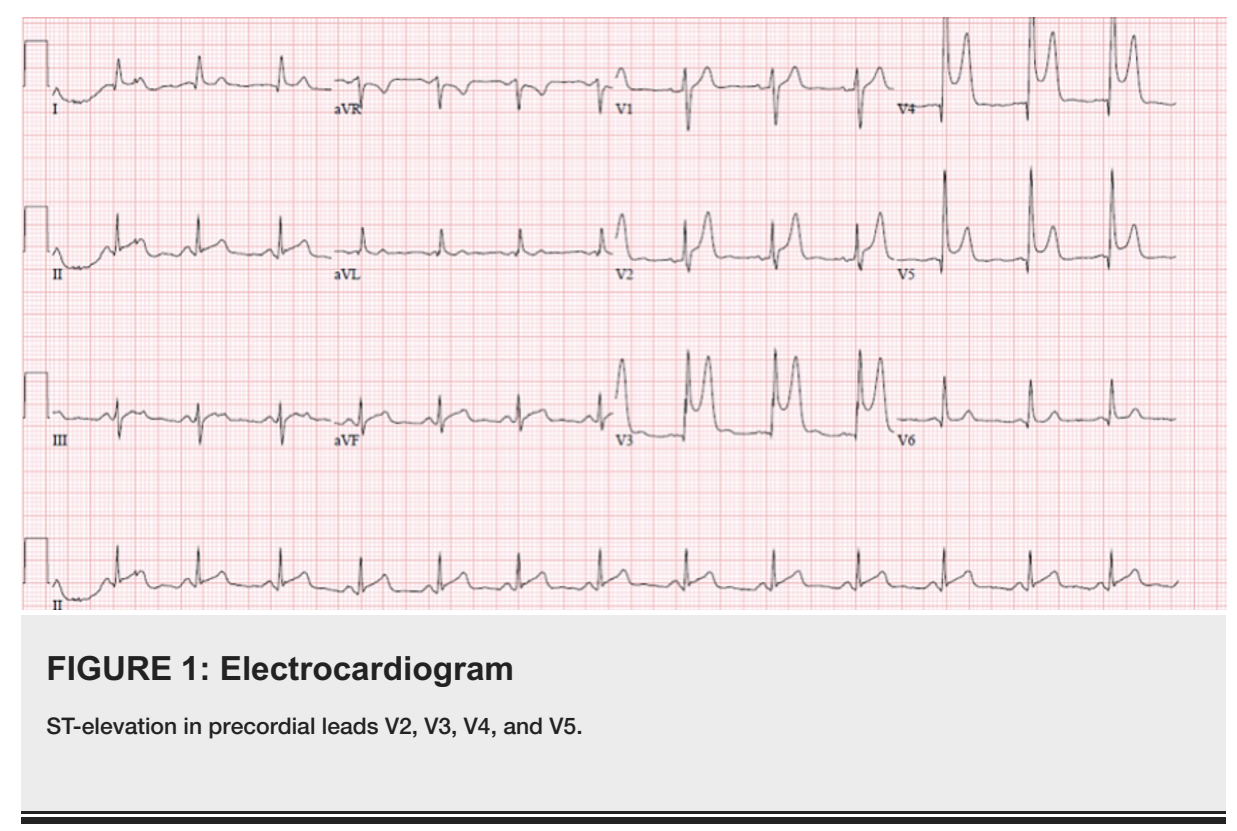

The ECG taken at the time of arrival in the emergency department revealed STEMI and elevated troponin. Cardiac catheterization revealed proximal left anterior descending artery $100 \%$ stenosis (Figure 2). A thrombectomy, percutaneous transluminal coronary angioplasty, and drug-eluting stent placement in the left anterior descending artery were performed. An ECG showed ischemic cardiomyopathy left ventricular ejection fraction $35-40 \%$, and apical and anterolateral wall hypokinesis. Systolic function was moderately reduced. There was mild hypokinesis of the mid-anteroseptal and mid-anteroseptal wall. There was akinesis of the apical anterior, apical septal, apical lateral, and the apical wall(s). Doppler parameters were consistent with abnormal left ventricular relaxation (grade 1 diastolic dysfunction). 


\section{Cureus}

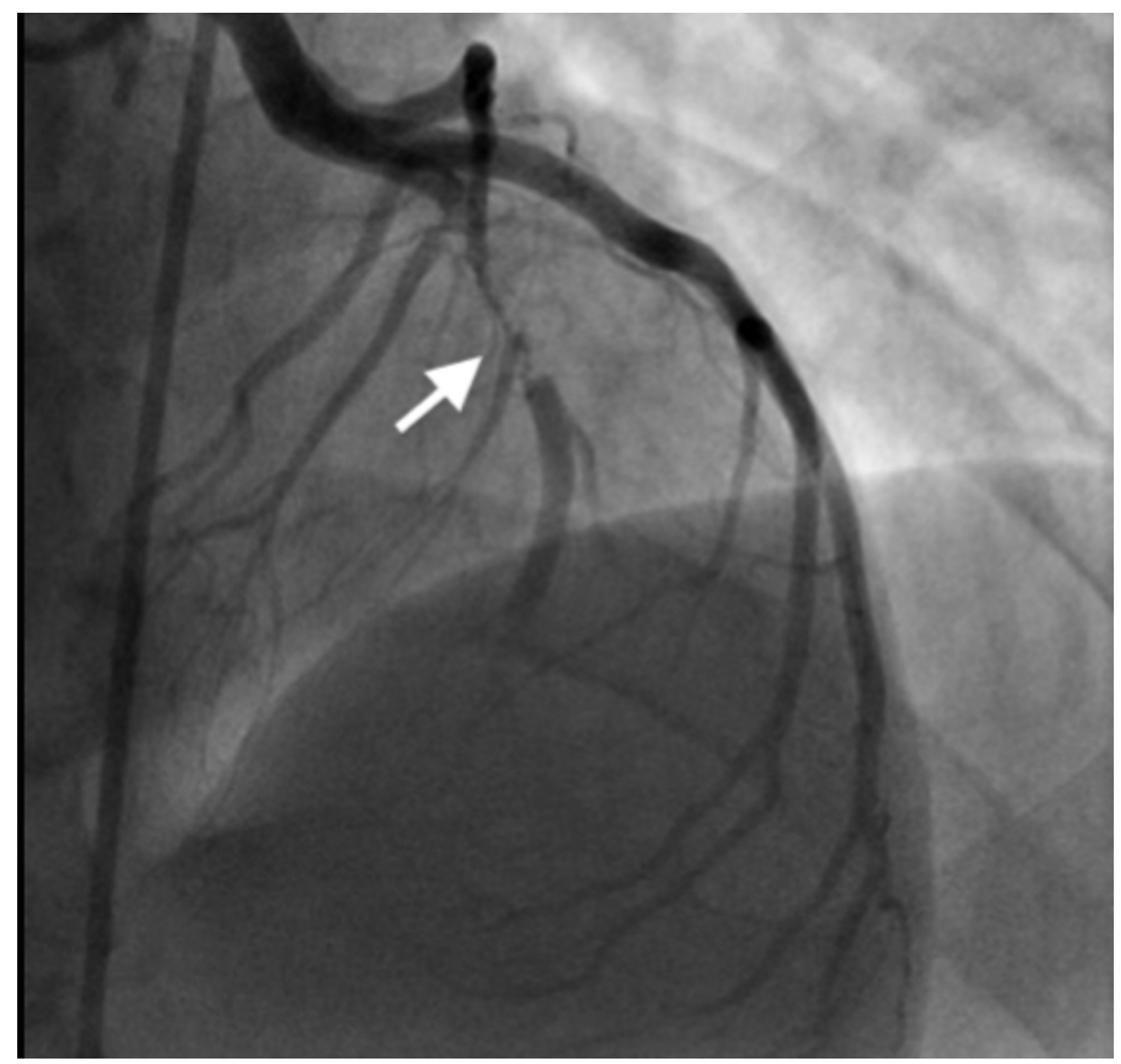

\section{FIGURE 2: Cardiac catheterization}

Proximal left anterior descending artery $100 \%$ stenosis.

All blood work including complete blood counts, chemistry, creatinine, liver enzymes, creatine kinase, homocysteine, vitamin B12, TSH, urine analysis, and urine drug screen was negative. The low-density lipoprotein (LDL) level $116 \mathrm{mg} / \mathrm{dl}$, high-density lipoprotein (HDL) $48 \mathrm{mg} / \mathrm{dl}$, triglycerides $141 \mathrm{mg} / \mathrm{dl}$, and total cholesterol $151 \mathrm{mg} / \mathrm{dl}$. The patient's coagulative workup including antithrombin III, factor V Leiden deficiency, protein $\mathrm{C}$ and $\mathrm{S}$ activity were within the normal range. Autoimmune workup was checked to rule out vasculitis including antinuclear antibody, antineutrophil cytoplasmic antibody, and anti-glomerular basement membrane antibody, and was negative.

The patient was stable during the hospital stay and asymptomatic. He was discharged on the third day of hospitalization. He was prescribed aspirin $81 \mathrm{mg}$ daily, ticagrelor $90 \mathrm{mg}$ daily, carvedilol $3.25 \mathrm{mg}$ twice daily, lisinopril $2.5 \mathrm{mg}$ daily, and atorvastatin $80 \mathrm{mg}$ daily. The aldosterone antagonist was not started inpatient secondary to borderline soft blood pressure. The patient was followed up as an outpatient in a week and his blood pressure was within normal limit, he was started on low-dose spironolactone $25 \mathrm{mg}$, and repeated basic metabolic was checked for potassium and that was within normal limit. The patient also had an echocardiogram after 40 days and his ejection fraction improved, and spironolactone was discontinued.

\section{Discussion}

Bodybuilders, weightlifters, and athletes frequently take AAS, a synthetic derivative of testosterone, in order to increase stamina and muscle mass [7]. Attaining a 10-20\% increase in strength of normal muscle mass is the main reason for abuse. Besides this positive aspect, there are a wide range of side effects, involving cardiovascular, cerebrovascular systems, and many more. The pathophysiology behind this is still unclear, but some hypotheses have been proposed. The prothrombotic effect of AAS is thought to be one mechanism. Steroids increase the synthesis of thromboxane A2 and decrease the synthesis of prostacyclin, thus leading to increased platelet aggregation. Also, increased thrombin activity contributes to a hypercoagulable state [3]. In addition, other hypothetical effects have been proposed such as impaired endothelial function and vasospasm. Steroid effects on homocysteine level causing hyperhomocysteinemia can lead to atherosclerotic and thrombotic effects in athletes. AAS can affect the absorption of B6 and B12 vitamins and cause an elevation in homocysteine levels [8]. 
Dyslipidemia and reduction in antioxidant levels are also reported as possible mechanisms. In a literature review, it was concluded that AAS have multiple effects on the cardiovascular system. Taking above the normal doses of AAS affect the heart structurally as well as functionally. The changes include myocardial hypertrophy, an increase in the diameters of heart chambers, and also marked changes in heart relaxation and contractile function. A temporary rise in blood pressure, prothrombotic effects, impaired lipid metabolism causing increase LDL and decrease HDL result in an increased risk of coronary artery disease. Ultimately AAS abusers can be at increased risk of life-threatening arrhythmias, leading to sudden cardiac death [3].

Athletes abusing AAS frequently attribute their symptoms to their workout and neglect minor symptoms of coronary artery disease and thus when they present to ER, they present with complications.

\section{Conclusions}

The young patient on anabolic steroid who presented to ED secondary to chest pain, such patients always need to work up for coronary artery disease. Athletes abusing AAS frequently attribute their symptoms to their workout and neglect minor symptoms of coronary artery disease and thus when they present to ER, they present with complications.

\section{Additional Information \\ Disclosures}

Human subjects: Consent was obtained or waived by all participants in this study. Conflicts of interest: In compliance with the ICMJE uniform disclosure form, all authors declare the following: Payment/services info: All authors have declared that no financial support was received from any organization for the submitted work. Financial relationships: All authors have declared that they have no financial relationships at present or within the previous three years with any organizations that might have an interest in the submitted work. Other relationships: All authors have declared that there are no other relationships or activities that could appear to have influenced the submitted work.

\section{References}

1. Kibble MW, Ross MB: Adverse effects of anabolic steroids in athletes . Clin Pharm. 1987, 6:686-692.

2. Du Toit EF, Rossouw E, Van Rooyen J, Lochner A: Proposed mechanisms for the anabolic steroid-induced increase in myocardial susceptibility to ischaemia/reperfusion injury. Cardiovasc J S Afr. 2005, 16:21-28.

3. Vanberg P, Atar D: Androgenic anabolic steroid abuse and the cardiovascular system. Handb Exp Pharmacol. 2010, 195:411-457. 10.1007/978-3-540-79088-4_18

4. Roe MT, Messenger JC, Weintraub WS, et al.: Treatments, trends, and outcomes of acute myocardial infarction and percutaneous coronary intervention. J Am Coll Cardiol. 2010, 56:254-263. 10.1016/j.jacc.2010.05.008

5. Poorzand H, Jafarzadeh Esfehani R, Hosseinzadeh P, Vojdanparast M: Acute myocardial infarction in a young male wrestler: a case report. ARYA Atheroscler. 2015, 11:366-369.

6. Coloma PM, Schuemie MJ, Trifirò G, et al.: Drug-induced acute myocardial infarction: identifying 'prime suspects’ from electronic healthcare records-based surveillance system. PLoS ONE. 2013, 8:e72148. 10.1371/journal.pone.0072148

7. Martinez-Quintana E, Saiz-Udaeta B, Marrero-Negrin N, Lopez-Mérida X, Rodriguez-Gonzalez F, Nieto-Lago $\mathrm{V}$ : Androgenic anabolic steroid, cocaine and amphetamine abuse and adverse cardiovascular effects . Int J Endocrinol Metab. 2013, 11:e8755. 10.5812/ijem.8755

8. Hartgens F, Kuipers H: Effects of androgenic-anabolic steroids in athletes. Sports Med. 2004, 34:513-554. 10.2165/00007256-200434080-00003 